\title{
A Balanced Virtual Machine Migration Mechanism based on Genetic Algorithm
}

\author{
Muhammad Abubakar ${ }^{1, a}$, Xu Youchang ${ }^{1, b}$, Yan Chengxin ${ }^{1, c}$ Chen Ningjiang ${ }^{1, d}$ \\ ${ }^{1}$ School of Computer and Electronic Information, Guangxi University, Nanning \\ aabubakar_90@hotmail.com, ${ }^{b} 18269009535 @ 163 . c o m, ~{ }^{c} y a n c h e n g x i n 313 @ 163 . c o m, ~{ }^{d} c h n j @ g x u . e$ \\ du.cn
}

Keywords: Virtual Machine Migration, Cloud Computing, Genetic Algorithm.

\begin{abstract}
A virtual machine allocation strategy based on genetic algorithm is proposed, which randomly selects a number of physical hosts as the initial population, and then calculates the fitness of each physical host. After applying the selection, crossover and mutation operator in physical population of each host, it builds the next generation of population, and cycles the above steps until reaching the termination conditions, thus the selected physical host will be allocated to the virtual machine. This method helps in load balancing optimization and ensures the stability and reliability of cloud services running with reducing the energy consumption and operating costs for data centers.
\end{abstract}

\section{Introduction}

Cloud computing using virtualization as a carrier which is very useful for scheduling computing power, network resources, and data storage. The real time migration technology of virtual machine is the most important and useful behavior in the field of virtualization, allowing virtual machines to be transferred from one physical host to another. The researchers now turn the research point to the virtual machine selection strategy and the virtual machine assignment strategy, with a view of reducing energy consumption in the data center and achieving greater load balancing for data centers.

\section{Related work}

Most common migration mechanisms include Pre-copy, Post-copy and some other hybrid techniques. Pre-copy is the most common approach for live migration of virtual machines. In order to control the downtime, the VM states are transferred to the destination in several iterations. It achieves very less downtime for the best case scenario but higher downtime because of stop-and-copy phase. Authors in [1] reduced the migration time by compressing the memory pages that are sent to destination in Pre-copy and stop-copy phase. The solution they proposed exploits the memory redundancy in order to gain compression ratios.

Authors in [2] proposed a pre-fetching technique in order to reduce as much as possible the migrated data simultaneously networks errors and faults, but it extends the total migration time. In literature [3] post copy was improved by adding a page fault filter which controls the migration process. Authors in [4] proposed a framework for estimating the duration of the overall process of change in migration and downtime. Other technologies include VMS [5] migrating from the source machine simultaneously to the target machine, Fast up and slow down (FUSD) [6] approach was developed to predict the rising trends of resources. This algorithm measures the CPU-workload at server and predicts the overloaded physical machine in the cloud platform.

\section{A VM migration Solution based on Genetic Migration}

In this paper, a strategy based on adaptive genetic algorithm [6] is designed towards VM migration. Genetic algorithm is based on the idea of genetics and natural selection, it can automatically acquire and guide the optimization of search space, and then adjust the search 
direction. The principle of the genetic algorithm is to select individuals from the current population as the first generation of genes, and use these selected ones to produce offspring.

Our idea is to look for the most effective scheduling solution in each scheduling performed by adaptive genetic algorithm which uses its genetic mechanism of survival of the fittest. According to the current states and already obtained historical data, genetic mechanism computes the influence which it will have when the current virtual machine resources.And assigns the virtual machine to the most adaptable physical host.

The genetic algorithm is characterized as follows:

A. In each iteration, the genetic algorithm starts to search from the initial value, thereby providing the optimum solution in the case of obtaining the optimum position.

B. Select the next group by using the computation of the random number generator.

C. Using genetic algorithms, the virtual machine load on each node is balanced (each physical machine has equal loads), and the system's load parameters are also obtained.

D. It has better optimization capability and internal implicit parallelism.

E. Selection rules used by genetic algorithms are probabilistic rather than deterministic selection rules.

The presented algorithm is described as follows.

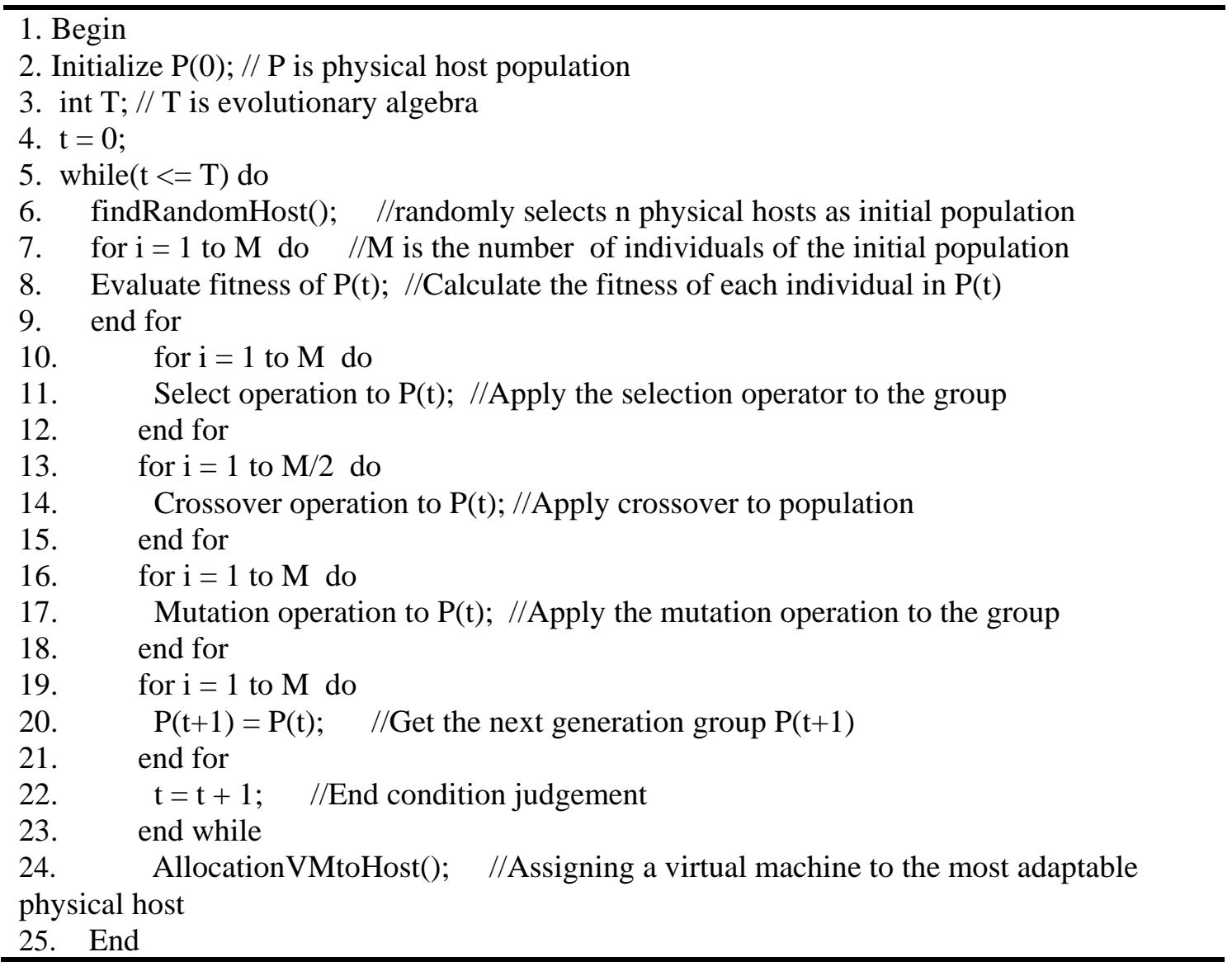

Experiments and Analysis. We use CloudSim framework [7] as simulation tool for validating the above algorithm. CloudSim aims to ease-up and speed the process of conducting experimental work which includes cloud computing as environment.

We carried out the experiments to test the performance and behavior of the presented mechanism. The testing machine is configured with the Windows 7 with minimum 4 cores of CPU, The least RAM storage of system is $8 \mathrm{~Gb}$.

Test of virtual machine migration success rate. A transaction is a unit of concurrency control, which is a user-defined sequence of operations. Vmware is selected as the platform to verify success 
rate. Through transactions, SQL Server binds a logically related set of operations together so that the server maintains the integrity of the data. Transactions of vmware are selected bdrv_set_dirty_tracking, set_dirty_bitmap, migrate_fd_put_ready, do_migrate and so on are compared to evaluate the success rate. Set_dirty_bitmap is used to set which sectors are dirty ,and migrate_fd_put_ready save the memory after the block has been migrated. Do_migrate complete the migration of the virtual machine,VM abnormal_end and Migrate abnormal_end finish the migration.Figure 1 shows the success rate of memory transaction verification for virtual machine online migration.

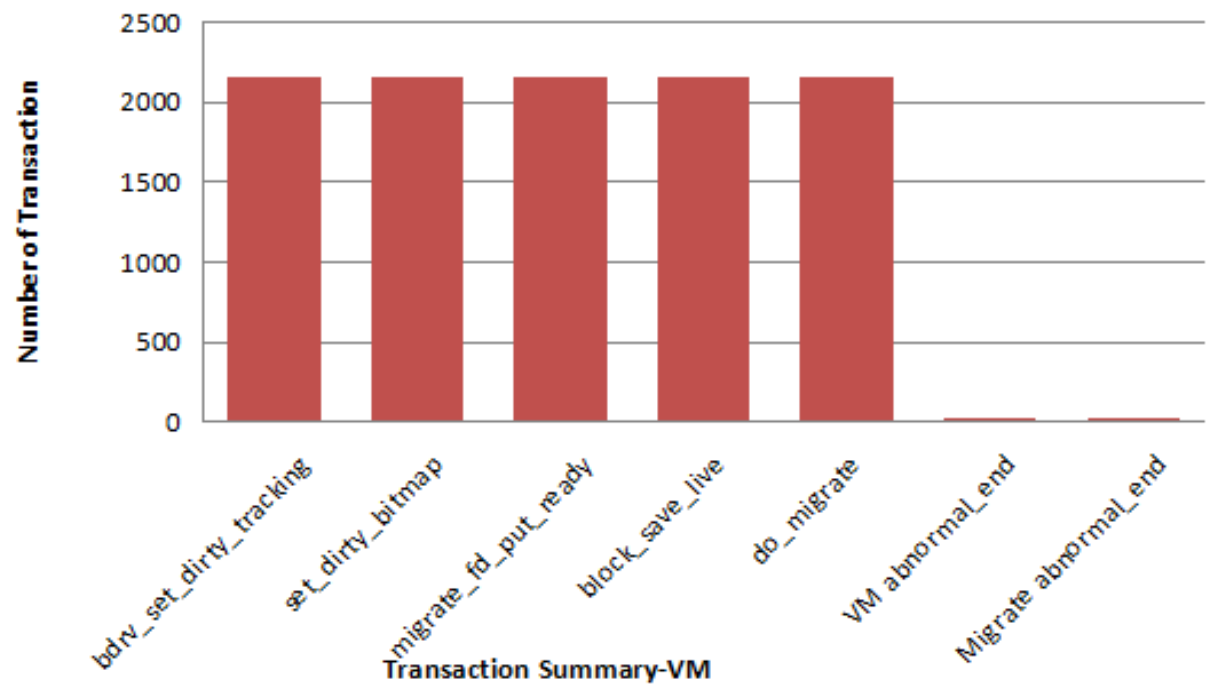

Figure 1 Transfer Success Rate

In addition to the success rate of bdrv_set_dirty_tracking and do_migrate, the success rate of transactions is high, which means that the finish number of transactions is 2163 , which indicates that all the online migration operations have been completed within 30 minutes. Then the success rate of the virtual machine continuous test is $100 \%$.

Resource utilization of online migration. This test is used to monitor the server's CPU utilization and memory usage. According to the experiments performed, average $75 \%$ of server resource utilization, using this algorithm greatly improves the performance of the virtual machine migration process. The result is shown in figure 2 and figure 3 . When we test the virtual machine online migration, we compared our algorithm with the FUSD algorithm mentioned in Section 2. We get the comparison graph from the data obtained by CloudSim simulation experiment.

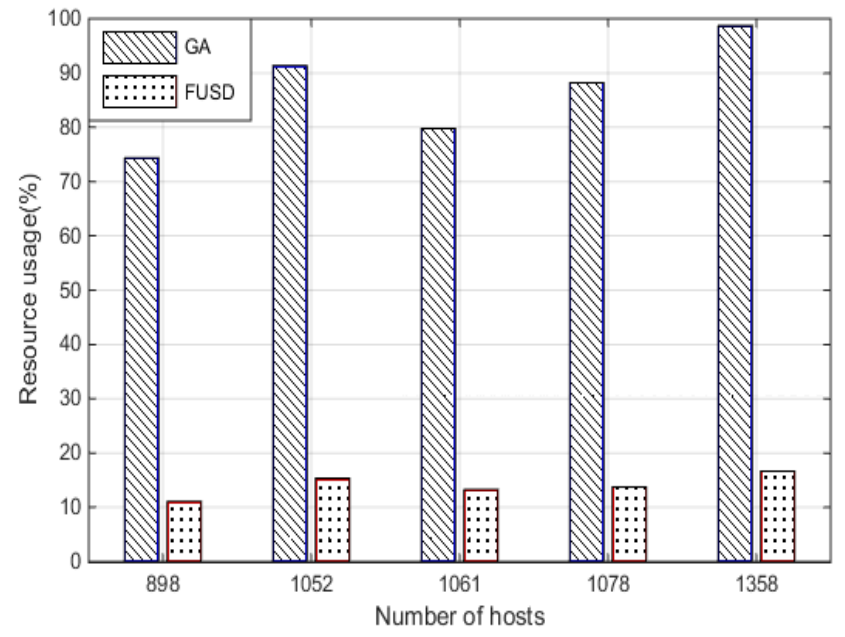

Figure 2 Resource utilization of Online migration

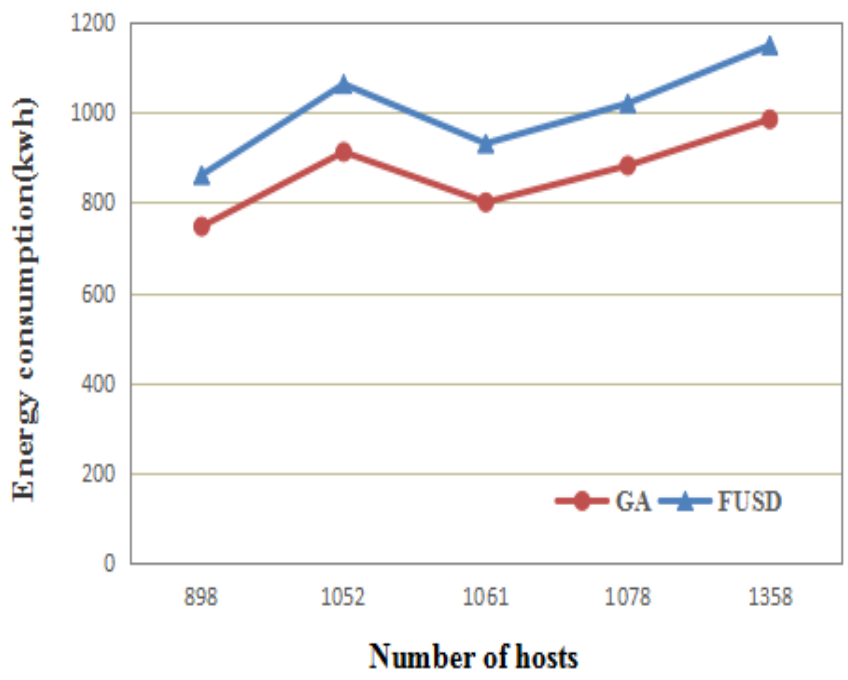

Figure 3 Comparison of energy consumption 
As shown in Figure 3, the number of analog hosts varies from 900 to 1400 units. The virtual machine migration algorithm using FUSD algorithm energy consumption between 600kwh to $1000 \mathrm{kwh}$, while using GA algorithm of power consumption at $100 \mathrm{kwh}$ to $150 \mathrm{kwh}$, it is known that GA based on the migration and distribution strategy of energy consumed less than based on the FUSD algorithm, thereby verifying the more efficiency of Genetic Algorithm when it comes to energy consumption.

In summary, the simulation results shows that the proposed algorithm can greatly improve the physical node load balancing in the virtual machine migration, with the minimum SLA violation rate and minimum energy consumption of cloud computing data center.

\section{Conclusions and Future Work}

A virtual machine migration and allocation strategy based on genetic algorithm is proposed in this paper,which reduces the energy consumption by reducing the number of migrations, and optimizes the load balancing of the data center. In the future work we will gather through the data, the optimum number of iterations is predicted by supervised learning.And a monitoring mechanism can also be introduced in order to further optimize the load balancing of data centers on migration.

\section{Acknowledgements}

This work is supported by the Natural Science Foundation of China(No. 61363003), the National Key Technology R\&D Program of China(No. 2015BAH55F02).

\section{References}

[1] Jin $\mathrm{H}$, Deng $\mathrm{L}$, Wu S, et al. Live virtual machine migration with adaptive, memory compression[C]// In Proceeding of the IEEE International Conference on CLUSTER Computing and Workshops. IEEE Xplore, 2009:1-10.

[2] M .R.Hines and K. Gopalan, "Post-copy based live virtual machine migration using adaptive pre-paging and dynamic self-ballooning," [C]// In Proceeding of the 2009 ACM SIGPLAN/SIGOPS international conference on virtual execution environments. ACM, 2009.

[3] Kui Su, Wenzhi Chen, Guoxi Li, Zonghui Wang, "RPFF: A Remote Page-fault Filter for Post-copy Live Migration” [C]//In Proceedings of the IEEE International Conference on Smart City/Social Com/ SustainCom together with DataCom, 2015.

[4] Akoush S, Sohan R, Rice A, et al. Predicting the Performance of Virtual Machine Migration[C]//In Proceedings of the IEEE International Symposium on Modeling, Analysis and Simulation of Computer and Telecommunication Systems. IEEE Computer Society, 2010:37-46.

[5] F. Checconi, T. Cucinotta, and M. Stein, "Real-time issues in live migration of virtual machines," [C]//In Proceedings of the Int. Conf. Parallel Proces, Workshops, 2010.

[6] Goldberg D E. The Existential Pleasures of Genetic Algorithms[J]. Cuesta Genetic Algorithms in Engineering \& Computer Science, 1994:23--31.

[7] Tian, Zhao, Xu, Zhung \& Sun “A Toolkit for Modeling and Simulation of Real Time Virtual Machine Allocation in a Cloud Data Center”, IEEE Transactions on Automated Science and Engineering, 2013. 